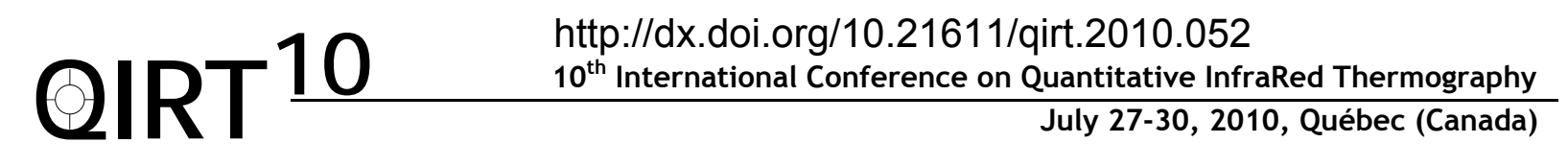

\title{
Wavelength-modulated differential photothermal radiometry for non-Invasive blood glucose detection
}

\author{
by X. Guo*, A. Mandelis", A. Matvienko, K. Sivagurunathan", and B. Zinman ${ }^{* *}$
}

\begin{abstract}
*Center for Advanced Diffusion-Wave Technologies, Mechanical \& Industrial Engineering, University Toronto, 5 King's College Road, Toronto, ON M5S 3G8, Canada

${ }^{\star *}$ Samuel Lunenfeld Research Institute, Mount Sinai Hospital, University of Toronto, 60 Murray Street, Toronto, ON M5T 3L9, Canada
\end{abstract}

Glucose detection is vital for diabetes management. The development of clinically viable noninvasive glucose biosensors has been hampered by lack of specificity and sensitivity. We introduce Wavelength-Modulated Differential Laser Photothermal Radiometry (WM-DPTR) for noninvasive blood glucose monitoring. WM-DPTR features unprecedented glucose-specificity and sensitivity by combining laser excitation by two out-of-phase modulated beams at wavelengths near the peak $(9.5 \mu \mathrm{m})$ and the baseline $(10.4 \mu \mathrm{m})$ of a prominent and isolated mid-IR glucose absorption band. Figure 1 shows the experimental set-up of the WM-DPTR system. Two out-of-phase modulated MIR laser beams (Laser A, $9.5 \mu \mathrm{m}$, and Laser $B, 10.4 \mu \mathrm{m}$ ), are steered onto a sample with a pair of flat mirrors. The generated two out-of-phase photothermal signals $S_{A} S_{B}$ are collected and focused onto the MCZT detector through a pair of off-axis paraboloidal mirrors. The signal from the detector is then sent to a lock-in amplifier for demodulation. The laser intensity ratio $I_{R}=I_{A} / I_{B}$ on the sample is strictly controlled with a pair of irises (Iris A and Iris B). Iris B is critical to the performance of the instrument and is motorized with diameter resolution $1.7 \mu \mathrm{m}$. The resulting differential signal $S_{A B}=S_{A}-S_{B}$ minimizes the background (water absorption/emission) effects, suppresses the overall signal range (from several hundred $\mu \mathrm{V}$ to several $\mu \mathrm{V}$ ) and is related to the glucose concentration of the sample with much higher sensitivity than single-ended signal $S_{A}$ or $S_{B}$. $S_{A B}$ can be expressed by two parameters: amplitude $A_{A B}$ and phase $P_{A B}$.

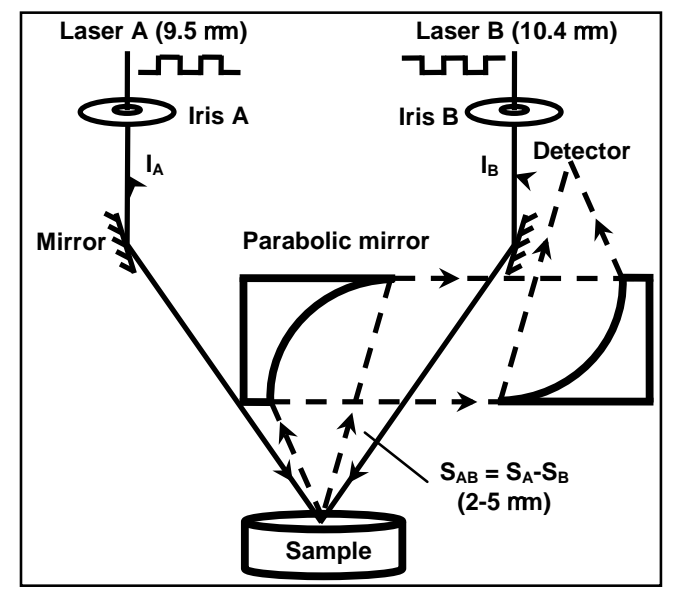

Fig. 1 Schematic diagram of the WM-DPTR system. Two out-of-phase modulated mid-IR laser beams (Laser A and Laser B) are steered by two flat mirrors onto a sample. The generated IR emission is collected and focused onto a MCZT ( $\mathrm{HgCdZnTe)}$ detector by a pair of parabolic mirrors. Laser intensity ratio $I_{R}$ on the sample is controlled by a pair of irises (Iris $A$ and Iris $B$ ).

Measurements on water-glucose phantoms $0-20 \mathrm{mmol} / \mathrm{l}(0-360 \mathrm{mg} / \mathrm{dl})$ demonstrate high sensitivity (up to $60 \%$ signal change due to $5.5 \mathrm{mmol} / \mathrm{l}(100 \mathrm{mg} / \mathrm{dl})$ change in glucose) and resolution $(0.07 \mathrm{mmol} / \mathrm{l}(1.3 \mathrm{mg} / \mathrm{dl}))$ to meet wide clinical detection requirements ranging from hypoglycemia to hyperglycemia. Fig. 2 is the measured WM-DPTR amplitude and phase vs. glucose concentration at intensity ratios $I_{R}=1.01,0.99$ and 0.98 and modulation frequency $49 \mathrm{~Hz}$. It is seen that both amplitude and phase can be used for optimally reliable glucose concentration diagnosis in a clinical setting. It is further observed that with decreasing intensity ratio, the sensitivity increases greatly at the expense of signal dynamic range. The highly sensitive measurement capability in low glucose concentration range is very attractive due to the lack of non-invasive methods for hypoglycemia monitoring below $3.85 \mathrm{mmol} / \mathrm{l}(70 \mathrm{mg} / \mathrm{dl})$ for adults and around $1.65-2.2 \mathrm{mmol} / \mathrm{l}(30-40 \mathrm{mg} / \mathrm{dl}) \mathrm{for}$ newborn infants. Shown in Fig. 3 are measurements at fixed laser intensity ratio $I_{R}=1.01$ and two modulation 

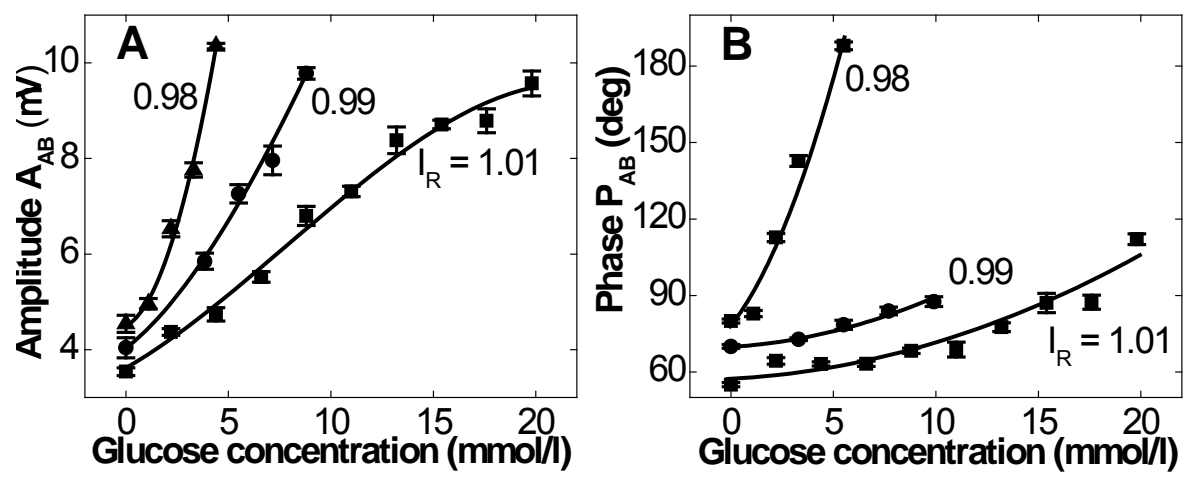

Fig. 2 Effect of laser intensity ratio on glucose measurement sensitivity and detection range at modulation frequency $f=$ $49 \mathrm{~Hz} . I_{R}=0.98,0.99$ and 1.01. (A) amplitude vs. glucose concentration. (B) phase vs. glucose concentration. Each datum is an average of five measurements. Changes in $I_{R}$ were made through fine adjustment of iris B, Fig. 1.

frequencies, $67 \mathrm{~Hz}$ and $20 \mathrm{~Hz}$. Both amplitude and phase are more sensitive at higher frequency. Fig. 3 also shows very complementary sensitivity to glucose between amplitude and phase across the full range of concentration.
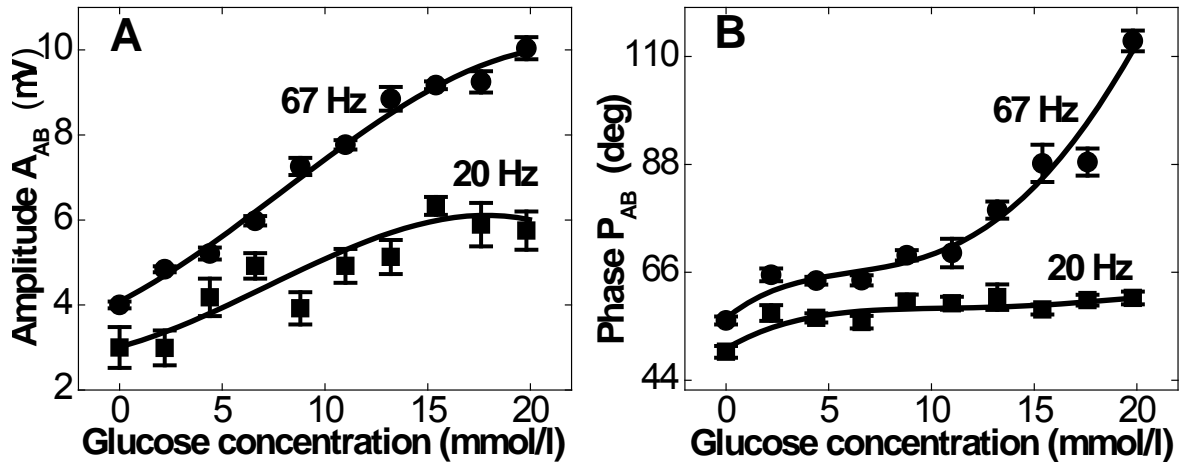

Fig. 3 Effect of modulation frequency on glucose measurement sensitivity at intensity ratio $I_{R}=1.01 . f=20 \mathrm{~Hz}$ and 67 $\mathrm{Hz}$. (A) amplitude vs. glucose concentration. (B) phase vs. glucose concentration. Each datum is an average of five measurements.

The glucose sensing capability of the WM-DPTR method is rooted in the optical and thermal property (absorption coefficient $\mu_{a}$ and thermal effusivity e) changes of the blood sample with glucose concentration. However, purely optical methods (such as transmission measurements) and purely thermal-wave methods (thermal effusivity measurements) are much less sensitive than WM-DPTR. This is so because the WM-DPTR method is based on the optical and thermal property change interdependence which greatly enhances differential signals: The thermal effusivity change acts as an amplifying factor of the optical absorption coefficient change. This is very important because optical changes add chromophore selectivity to WM-DPTR, whereas thermal changes alone are not selective at a molecular level.

We have introduced WM-DPTR for glucose detection in aqueous phantoms in the clinically relevant range. The amplitude and phase of the WM-DPTR signal act as two complementary glucose metrics and yield reliable results. Through proper selection of the excitation laser intensity ratio and the corresponding optimal modulation frequency, the WM-DPTR glucose measurement mode can be adjusted for maximum sensitivity to the glucose range of interest for accurate evaluation of biologically relevant glucose concentrations, from hypoglycemia to hyperglycemia. In practice, glucose detection instrumentation based on WM-DPTR is able to perform preliminary (coarse) measurements in the maximum dynamic range and relatively low sensitivity mode to locate a patent's glucose concentration range and then use appropriate intensity ratio to switch to the high-resolution mode for precise glucose measurements. 